\title{
Coronavirus Disease-2019 and Mental Health
}

\author{
Riska Habriel Ruslie ${ }^{1}$, Darmadi Darmadi ${ }^{2 *}$ \\ ${ }^{1}$ Department of Child Health, Faculty of Medicine, Universitas Prima Indonesia, Medan, Indonesia; ${ }^{2}$ Department of Internal \\ Medicine, Faculty of Medicine, Universitas Sumatera Utara, Medan, Indonesia
}

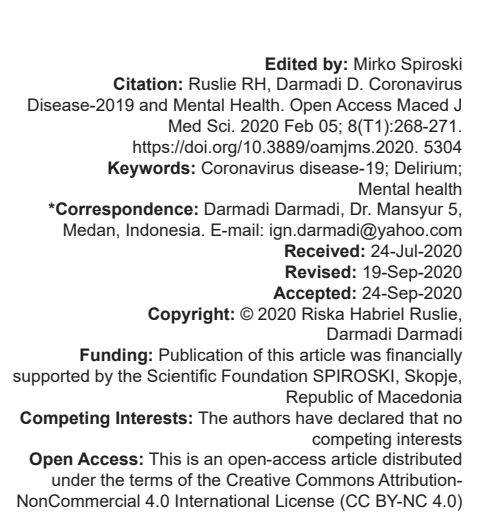

Introduction

Emerging in Wuhan, China in the end of 2019, coronavirus disease-2019 (COVID-19) has spread throughout the globe and caused significant morbidity and mortality [1], [2]. The World Health Organization had finally declared COVID-19 as a pandemic on March 11, 2020 [3]. There are many manifestations of COVID-19, including respiratory and gastrointestinal symptoms, but only a little attention is paid for mental health problem [1], [3], [4]. Before this COVID-19 pandemic, other coronaviruses had already caused an epidemic which is a severe acute respiratory syndrome (SARS) in 2002 and Middle East respiratory syndrome (MERS) in 2012 [4]. Previous coronavirus epidemics also triggered mental health problems. The deficit in neuropsychiatry was reported to be present until 18 months post-discharge [5]. During the previous SARS epidemic, $59 \%$ of SARS survivors suffered from psychiatric disorders in the first 3 years [1].

This COVID-19 pandemic is different with other disasters such as earthquake or tsunami. This pandemic showed no sign of ending. Besides, this pandemic affects everyone in every moment and every place. This causes a big uncertainty in one's mind and leads to the mental problem [6]. It is important to note that mental health disturbance can cause more significant effects than the virus itself [7]. Several literatures had described mental health problems in association with COVID-19. A patient with COVID-19 was suffering from meningoencephalitis, marked by neck stiffness and transient generalized seizure [1]. Another study reported that $31 \%$ of patients with COVID-19 had altered mental status. Mental health problems seem to occur more often in younger patients [2]. Beach et al. reported 4 case series of COVID-19 patients with delirium [8]. There was also a positive association observed between coronavirus seropositivity and mood disorders [1]. A study from Egypt reported a significant psychological impact in the public from COVID-19 pandemic with depression as the most frequent, followed by anxiety [9].

\section{Methods}

We gathered literature regarding the topics from electronic sources, including PubMed and Google Scholar. We used "coronavirus," "coronavirus disease 2019," "COVIC-19," "mental," "health," "disorder," and "disturbance" as the keywords in the search engine. We included literature published from December 2019 and on. All literatures were written in English. 


\section{Discussion}

\section{Pathogenesis of mental health disturbance in COVID-19}

Coronavirus is a single-stranded ribonucleic acid virus with four major genera: Alphacoronavirus, betacoronavirus, gammacoronavirus, and deltacoronavirus. Previous epidemics, SARS-CoV, and MERS-CoV, together with SARS-CoV-2, belong to betacoronavirus genera [1], [4], [10]. SARS-CoV-2 particles are detected in patients with multiple sclerosis giving a higher possibility of the virus neurotropism [1]. In vitro study had shown that SARS-CoV-2 can replicate in neurons [4], [5].

SARS-CoV-2 is hypothesized to enter the brain through the olfactory bud, resulting in rapid invasion with transneuronal spread and minimal cellular infiltration. This explains the presence of anosmia in patients with COVID-19 [1], [5], [8], [10], [11]. Viral invasion may occur through binding of virus with angiotensin-converting enzyme 2, which expressed in neurons, capillary, and neuronal endothelium. After invasion, the virus will trigger an inflammatory reaction, create cytokine storm [4], [5], [8], [10], [11], and lead to neuropsychiatric symptoms. Invasion into postrema increases the vulnerability of subjects toward depression and delirium [1], [5], [12]. Virus-induced central nervous system demyelination also occurs. Demyelination occurs due to an inflammatory reaction from molecular mimicry between coronavirus envelope $S$ glycoproteins and myelin [1], [5], [12]. Other causes of mental disorders, including secondary to multiorgan failure, hypoxia, and treatmentrelated [4], [8], [11], [12]. In addition, possible medullary neurons destruction after viral invasion may precipitate respiratory symptoms and cause acute respiratory distress [5], [8], [11]. Stress activates hypothalamuspituitary-adrenal axis and impairs immune system which further hampers the clinical condition [11].

In this modern era, every subject has the capability to travel and communicate widely. At present, these freedoms are restricted significantly due to COVID-19 [3], [13]. This condition affects both healthy and infected population [4] and leads to frustration and mental problems [3], [4], [5], [11], [13]. Hospitalization and uncertain prognosis of COVID-19 also clearly worsen the mental disorders [1], [5], [13]. Adverse events from medications used in COVID-19 contribute to mental health status such as insomnia from corticosteroid and psychotic disorder from chloroquine [1], [5], [11]. There is a hypothesis that SARS-CoV-2 may induce vasculopathy and coagulopathy. If the conditions occur in the brain, neuropsychiatry syndromes may manifest [2]. Excessive use of the internet during isolation also contributes to impaired mental function, mainly through the spread of unverified information regarding COVID19 [7], [6], [13]. Risk factors contributing to a mental health problem in COVID-19 patients are female gender, poor-self-related health, and the presence of relatives with COVID-19 [10]. Inadequacy of basic supplies and unclear information regarding the ongoing situation is considered as additional risk factors [13].

\section{The symptoms of mental health disturbance}

A wide range of mental health symptoms is observed, including anxiety, fear, loneliness, anger, encephalopathy, delirium, congenital impairment, mood swings, insomnia, suicide, psychosis, and general distress [1], [4], [6], [7], [11], [13]. Loss of significant ones may also result in persistent yearning and symptoms of reactive distress and social/identity disruption causing impairment in daily life. This condition is known as persistent complex bereavement disorder [14]. Mental health symptoms may manifest even in the absence of respiratory symptoms [12]. The impact of COVID-19 to mental health is always neglected in the acute phase of the disease. It is due to the critical course of COVID-19 which needs more attention and prompt management [3], [6]. Around $35 \%$ patients with COVID-19 were reported to suffer from psychological distress [6]. Patients with COVID-19 had a higher rate of suffering from post-traumatic stress disorder (96.2\%) and depression (29.2\%) [10]. Other studies reported that $65 \%$ and $69 \%$ of COVID-19 patients who admitted to intensive care unit had delirium and agitation, respectively. Altered consciousness was reported in $21 \%$ of patients who deceased [4]. Approximately one-third of COVID-19 patients had neuropsychiatric syndromes such as encephalopathy and altered consciousness [8]. Patients with underlying mental problems have a higher risk in developing more severe symptoms; therefore, they should be treated with antipsychotic drugs together with standard COVID-19 treatment [6], [10].

The symptoms are not only present during the disease course but also after the patients recovered. Lower psychological well-being and higher anxiety score are observed in patients survived from COVID19 [10]. At discharge, $14.8 \%$ of patients had anxiety, while $14.9 \%$ and $32.2 \%$ had depression and posttraumatic stress disorder, respectively [4]. Another study also reported mental health disorders such as post-traumatic stress disorder $(54.5 \%)$, depression $(39 \%)$, pain disorder $(36.4 \%)$, panic disorder $(32.5 \%)$, and obsessive-compulsive disorder (15.6\%). This urges sustained follow-up for the mental health status of post-discharged patients [5].

\section{Mental Health Problem in Health-care Personnel}

Mental health problems are also found in health-care personnel in intense contact with COVID-19 
patients, particularly general practitioners in emergency and staffs in intensive care units. They usually have fear, psychological distress, burnout, anxiety, depression, insomnia, somatization, post-traumatic stress disorder, and obsessive-compulsive disorders [5], [6], [10], [15]. The identified etiologies for a mental health problem in health-care personnel are lack of personal protective equipment, high working pressure, suboptimal training/ confidence when working in extreme circumstances, concerns of being infected or infecting their relatives, and lack of adequate support in the working environment [15].

\section{Diagnosis of mental health problem}

Before diagnosing COVID-19 patients with a mental disorder, we must exclude other possibilities such as metabolic disorders, hydration status, and medication effect. Brain magnetic resonance imaging (MRI) of COVID-19 patients with neuropsychiatric syndrome showed leptomeningeal enhancement and bilateral frontotemporal hypoperfusion. EEG finding is not specific, showing diffuse background slowing consistent with encephalopathy [8], [12]. Cerebrospinal fluid examination should be conducted in patients with seizure, encephalitis, and encephalomyelitis as coronaviruses may be detected [4]. After those possibilities have been excluded, we can assess one's mental health status using several assessment tools. The Mental Health Quotient can be applied. It is safe enough since it is a web-based assessment tool. It is also fast, easy, and comprehensive toll based on Diagnostic and Statistical Manual of Mental Disorders (DSM)-5 [16]. However, DSM-5 is still being the most common and standard assessment tool for mental disorder used at present [17].

\section{Treatment of mental health problems in COVID-19 patients}

Literature regarding mental health problems due to COVID-19 and its management is scarce [3]. Psychological service should be maintained to prevent the effect of COVID-19 to patient's mental health. Effective education and communication are important along with the management of stigma and discrimination toward patients [3], [13]. Constructive peer support significantly aids the management of mental health problems [7]. Other suggestions regarding calming skills, health and optimism maintenance, and social support system are also important in keeping the wellness of mental health [3], [12]. Cognitive-behavioral therapy (CBT) may be implemented in patients with mental health problems. It is suspected to be able to relieve the symptoms of mental disorder. During the pandemic, online CBT is one of the safest options [14]. Patients should have access to communication devices such as phone and tablet and also early ambulation. If behavioral management does not improve the symptoms, pharmacological management should be started [12]. Medications used to manage mental health problems in COVID-19 patients are antipsychotic agents (olanzapine and chlorpromazine), haloperidol, antidepressants, benzodiazepines, and amantadine [8], [11]. Melatonin, alpha-2 agonist, valproic acid, and dopamine agonists are also administered in patients with delirium. Vitamins are not a standard treatment for delirium. However, they have antioxidant and anti-inflammatory effects, which, more or less, may protect against neuropsychiatric symptoms [12]. Further study regarding the efficacy of each medication is mandatory [8], [11].

\section{Conclusion}

Mental health problems are frequently found in patients with COVID-19. The pathogenesis or mental health problems rely on direct invasion of virus, inflammation-induced demyelination, secondary to multiorgan failure, hypoxia, treatment-related, isolation, unconfirmed information, coagulopathy, vasculopathy, and uncertain outcome. Its symptoms vary widely, including delirium and anxiety. The symptoms are reported in both patients and health-care personnel. The auxiliary examinations for its diagnosis are not specific. Its management consists of psychological and medical management.

\section{References}

1. Khateb M, Bosak N, Muqary M. Coronaviruses and centra nervous system manifestations. Front Neurol. 2020;11:715. https://doi.org/10.3389/fneur.2020.00715 PMid:32655490

2. Varatharaj A, Thomas N, Ellul MA, Davies NW, Pollak TA Tenorio EL, et al. Neurological and neuropsychiatric complications of COVID-19 in 153 patients: A UK-wide surveillance study. Lancet Psychiatry. 2020;7(10):875-82. https://doi.org/10.2139/ssrn.3601761

3. Ransing $R$, Adiukwu $F$, Pereira-Sanchez V, Ramalho $R$, Orsolini L, Teixeira AL, et al. Mental health interventions during the COVID-19 pandemic: A conceptual framework by early career psychiatrists. Asian J Psychiatr. 2020;51:102085. https:// doi.org/10.1016/j.ajp.2020.102085

PMid:32413616

4. Rogers JP, Chesney E, Oliver D, Pollak TA, McGuire P, FusarPoli $\mathrm{P}$, et al. Psychiatric and neuropsychiatric presentations associated with severe coronavirus infections: A systematic review and meta-analysis with compatison to the COVID-19 pandemic. Lancet Psychiatry. 2020;7(7):611-27. https://doi. org/10.1016/s2215-0366(20)30203-0

PMid:32437679

5. Troyer EA, Kohn JN, Hong S. Are we facing a crashing wave of neuropsychiatric sequelae of COVID-19? Neuropsychiatric symptoms and potential immunologic mechanisms. Brain Behav 
Immun. 2020;87:34-9. https://doi.org/10.1016/j.bbi.2020.04.027 PMid:32298803

6. Giallonardo V, Sampogna G, Vecchio VD, Luciano M, Albert U, Carmassi $\mathrm{C}$, et al. The impact of quarantine and physical distancing following COVID-19 on mental health: Study protocol of a multicentric Italian population trial. Front Psychiatry. 2020;11:533. https://doi.org/10.3389/fpsyt.2020.00533

PMid:32581895

7. Kontoangelos K, Economou M, Papageorgiou C. Mental health effects of COVID-19 pandemia: A review of clinical and psychological traits. Psychiatry Investig. 2020;17(6):491-505. https://doi.org/10.30773/pi.2020.0161

PMid:32570296

8. Beach SR, Praschan NC, Hogan C, Dotson S, Merideth F, Kontos $\mathrm{N}$, et al. Delirium in COVID-19: A case series and exploration of potential mechanisms for central nervous system involvement. Gen Hosp Psychiatry. 2020;65:47-53. https://doi. org/10.1016/j.genhosppsych.2020.05.008 PMid:32470824

9. Arafa A, Mohamed A, Saleh L, Senosy S. Psychological impacts of the COVID-19 pandemic on the public in Egypt. Community Ment Health J. 2020;2020:1-6. https://doi.org/10.1007/ s10597-020-00701-9 PMid:32803445

10. Vindegaard N, Benros ME. COVID-19 pandemic and mental health consequences: Systemic review of the current evidence. Brain Behav Immun. 2020. https://doi.org/10.1016/j. bbi.2020.05.048

PMid:32485289

11. Dinakaran D, Manjunatha N, Kumar CN, Suresh BM. Neuropsychiatric aspects of COVID-19 pandemic: A selective review. Asian J Psychiatr. 2020;53:102188. https://doi. org/10.1016/j.ajp.2020.102188

PMid:32512530

12. Baller EB, Hogan CS, Fusunyan MA, Ivkovic A, Luccarelli JW, Madva E, et al. Neurocovid: Pharmacological recommendations for delirium associated with COVID-19. Psychosomatics. 2020. https://doi.org/10.1016/j.psym.2020.05.013 PMid:32828569

13. Serafini G, Parmigiani B, Amerio A, Aguglia A, Sher L, Amore M. The psychological impact of COVID-19 on the mental health in the general population. QJM. 2020;113(8):531-7. https://doi. org/10.1093/qjmed/hcaa201

14. Lenferink L, de Keijser J, Eisma M, Smid G, Boelen P. Online cognitive-behavioural therapy for traumatically bereaved people: Study protocol for a randomized waitlist-controlled trial. BMJ Open. 2020;10(9):e035050. https://doi.org/10.1136/ bmjopen-2019-035050

PMid:32883723

15. de Pablo GS, Vaquerizo-Serrano J, Catalan A, Arango C, Moreno C, Ferre F, et al. Impact of coronavirus syndromes on physical and mental health of health care workers: Systematic review and meta-analysis. J Affect Disord. 2020;275:48-57. https://doi.org/10.1016/j.jad.2020.06.022 PMid:32658823

16. Newson JJ, Thiagarajan TC. Assessment of population wellbeing with the mental health quotient (MHQ): Development and usability study. JMIR Ment Health. 2020;7(7):e17935. https:// doi.org/10.2196/17935

17. Stein DJ, McLaughlin KA, Koenen KC, Atwoli L, Friedman MJ, Hill ED, et al. DSM-5 and ICD-11 definitions of posttraumatic stress disorder: Investigating "narrow" and "broad" approaches. Depress Anxiety. 2014;31(6):494-505. https://doi.org/10.1002/ da. 22279

PMid:24894802 